\title{
Effects of Astragalus fasciculifolius gum on wound healing in streptozotocin-induced diabetic rats
}

\author{
Mohammadali Ayubi-Rad ${ }^{\mathbb{1}}$, Soheila Yosefi ${ }^{1}$, Mohammadreza Hajizadeh $^{2,3}$, Hamidreza Jafari-Naveh $^{4}$, Mahsa $^{2}$ \\ Hassanipour ${ }^{5,6}$, Fatemeh Alamchi ${ }^{1}$, Afsaneh Fadai ${ }^{1}$, Faridehsadat Khorasani ${ }^{1}$, Farzanehsadat Hosseini ${ }^{2}$, Mahdieh \\ Bemani $^{2}$, Mehdi Mahmoodi ${ }^{* \mathbb{D}}$ \\ ${ }^{1}$ Department of Laboratory Sciences, Faculty of Paramedicine, Rafsanjan University of Medical Sciences, Rafsanjan, Iran \\ ${ }^{2}$ Department of Clinical Biochemistry, Faculty of Medicine, Rafsanjan University of Medical Sciences, Rafsanjan, Iran \\ ${ }^{3}$ Molecular Medicine Research Center, Research Institute of Basic Medical Sciences, Rafsanjan University of Medical Sciences, Rafsanjan, Iran \\ ${ }^{4}$ Department of Anatomy, School of Medicine, Rafsanjan University of Medical Sciences, Rafsanjan, Iran \\ ${ }^{5}$ Physiology-Pharmacology Research Center, Research Institute of Basic Medical Sciences, Rafsanjan University of Medical Sciences, Rafsanjan, Iran \\ ${ }^{6}$ Department of Physiology and Pharmacology, School of Medicine, Rafsanjan University of Medical Sciences, Rafsanjan, Iran \\ ${ }^{7}$ Department of Clinical Biochemistry, Afzalipoor Faculty of medicine, Kerman University of Medical Sciences, Kerman, Iran
}

\section{A R T I C L E I N F O}

Article Type:

Original Article

\section{Article History:}

Received: 27 October 2018

Accepted: 17 May 2019

\section{Keywords:}

Medicinal plant

Diabetic wound

Astragalus fasciculifolius

Streptozotocin

Rat

\begin{abstract}
A B S T RACT
Introduction: Recently it has been shown that Astragalus fasciculifolius may be an effective remedy in the treatment of diabetes and wound healing. Current study was designed to examine the effects of topical administration of $A$. fasciculifolius (common name: sarcocolla) cream on treatment of wounds in streptozotocin-induced diabetic rats.

Methods: A circle deep wound having $15-\mathrm{mm}$ diameter was created on the back of 32 streptozotocin-induced diabetic rats. Thirty-two wounds were evaluated in four groups of animals $(\mathrm{n}=8)$. The animals in first and second groups were treated using distilled water and petrolatum for 20 days, respectively. The third and fourth groups were treated using sarcocolla cream $5 \%$ and $10 \%$ for 20 days, respectively. The evaluation was performed in all groups on days $1,3,5,7,10,14,17$ and 20 .

Results: The sarcocolla cream $5 \%$ and sarcocolla cream $10 \%$ exerted significant healing effects on diabetic rat wounds $(P<0.001)$ compared to the control groups under treatment of petrolatum or distilled water. The sarcocolla cream had favorable effects on the woundhealing ratio especially during long-term (14 days), with no difference at concentrations of sarcocolla $5 \%$ and $10 \%$.

Conclusion: Sarcocolla (topical) therapy is possibly a useful approach for wound healing in diabetic rats. More investigations are needed to elucidate the possible protective mechanisms and safety.
\end{abstract}

Implication for health policy/practice/research/medical education:

This study revealed the protective effects of Astragalus fasciculifolius gum on wound healing in streptozotocin-induced diabetic rats. This possibly safe herbal remedy will be a potential therapeutic approach for the common complication of diabetes mellitus in future after elucidating the mechanism of action.

Please cite this paper as: Ayubi-Rad M, Yosefi S, Hajizadeh M, Jafari-Naveh H, Hassanipour M, Alamchi F, et al. Effects of Astragalus fasciculifolius gum on wound healing in streptozotocin-induced diabetic rats. J Herbmed Pharmacol. 2020;9(4):328332. doi: $10.34172 /$ jhp.2020.41.

\section{Introduction}

Diabetes mellitus is one of the most common kind of glandular disorder in the world and the most common cause of blindness, kidney diseases and lower-limb amputation (1). Common symptoms of diabetes include frequent urination, increased water and food intake, weight loss, restlessness and increased thirst (2).
Chronic complications of diabetes include retinopathy, nephropathy, cardiovascular disease, and skin diseases such as diabetic wounds (3). These wounds are slow healing wounds that can continue for weeks despite the appropriate care (4). Diabetes related wounds are considered as serious complications of diabetes due to the high frequency and impaired healing which lead to chronic wounds and 
amputation (4). Evidence from animal and human studies showed several abnormalities in multiple stages of the wound healing process (5). Mechanisms of poor and slow wound healing in diabetic patients may include disruption of cytokine production and repair factors (6), abnormal angiogenesis $(7,8)$, and reduced neuropeptide levels (9). One of the main goals of researchers and surgeons in this field is to achieve an approach with maximum efficacy and minimum side effects that accelerates the healing of the wounds. Following these goals, natural chemicals and medicinal herbs are an interesting target for detecting a new therapy in diabetic wound management $(10,11)$. Modern treatment of diabetic wounds consists of blood glucose control, wet dressings and systemic antibiotics (12). Among the different herbal remedies, Astragalus fasciculifolius (common name: sarcocolla/Family: Fabacea) has been considered as an effective treatment. Studies have shown anti-diabetic and wound healing effects for this herb $(13,14)$. As there is not enough data about the beneficial properties of Astragalus fasciculifolius on diabetic wounds, in this study we investigated the possible healing effect of $A$. fasciculifolius gum effect on the wounds of diabetic rats.

\section{Materials and Methods}

\section{Animals}

In this study 32 Albino Wistar rats weighing 200-250 g were used. Animals were housed in polycarbonate cages (temperature: $25 \pm 2^{\circ} \mathrm{C}$, 12 hour light/12 hour dark cycle). The rats were given standard pellet chow and tap water $(15,16)$. The protocol was confirmed in Ethical Committee of Rafsanjan University of Medical Sciences (Ethical code: IR.RUMS.REC.1397.078).

\section{Preparation of cream}

Astragalus fasciculifolius cream was formulated using gum and petrolatum. Plant was obtained from the herbal market in Rafsanjan, Iran. The plant was identified in the department of biochemistry in Rafsanjan University of Medical Sciences, Iran. Plant materials (gum) were cleaned to remove the dirt and extra material and were ground to a coarse powder using a laboratory mill. Powder was dissolved in warm distilled water $\left(45-55^{\circ} \mathrm{C}\right)$ and then mixed with the base of cream during cream forming to create concentrations of $5 \%$ and $10 \%$ (17).

\section{Induction of diabetes with streptozotocin}

In order to induce diabetes in rats an intraperitoneal (i.p.) injection of streptozotocin with the dose of $50 \mathrm{mg} /$ $\mathrm{kg}$ prepared in low temperature normal saline was used (18). Three days after the injection, hyperglycemia was confirmed by measuring the blood glucose of rats using glucometer (blood was collected from the end of their tails). Animals showing fasting blood sugar levels more than $200 \mathrm{mg} / \mathrm{dL}$ were considered to be diabetic (19).
Induction and treatment of skin wound

The diabetic rats were all anesthetized by inhaling ether. The hair on their back (dorsum) was shaved and wound field was outlined with a marker before removing the skin. A circled field having $1.5 \mathrm{~cm}(15 \mathrm{~mm})$ diameter was drawn on their dorsum by marker. By using the appropriate surgical equipment, a deep skin wound was created via removing a circle of skin with $15 \mathrm{~mm}$ diameter on their dorsum while removing the subcutaneous connective tissue.

Thirty-two wounds were studied in four groups (each group: $\mathrm{n}=8$ ). The first and second groups were treated using distilled water and petrolatum for 20 days, respectively. The third and fourth groups were treated using sarcocolla cream $5 \%$ and $10 \%$ for 20 days, respectively. Before treatment, all wounds were cleaned daily with sterile saline. Then, the creams were administered equally in amounts sufficient for covering the entire wound area. Treatments were applied daily around 5-6 PM with the procedure of taking pictures (20-22).

\section{Healing evaluation}

After treatment initiation in appropriate time points (days 1, 3, 5, 7, 10, 14 and if not cured 17 and 20); the wound healing was evaluated by measurement of wound surface area using caliper Vernier and the ImageJ software. Finally, healing ratio was calculated by this equation $(21,23)$ :

\section{Healing ratio $(\%)=100 \times(1-$ wound area after treatment/ initial wound area)}

\section{Statistical analysis}

Data were analyzed by two-way repeated measures ANOVA with Bonferroni post hoc and Tukey's tests. SPSS version 21.0 for Windows was used for statistical analysis. The level of significance was $P<0.05$.

\section{Results}

Two-way repeated measures ANOVA showed that the time effect (days 1, 3, 5, 7, 10, 14, 17 and 20) was statistically significant $(P<0.001)$, meaning that, regardless to the drug treatment in different groups, with increasing days, the average wound healing percentage in rats significantly changes and evaluation time plays an important role in the increase of healing ratio percentage.

The results of Bonferroni post hoc test also showed that regardless of the drug treatment in different groups, the average of healing of the wound surface in rats that were compared two by two was significantly different in all eight evaluation time (day 1, 3, 5, 7, 10, 14, 17 and 20) $(P<0.001)$.

The above test also showed that regardless of the evaluation time the effect of drug in different treatment 
groups was also significant, meaning that, regardless of evaluating the time, the average wound healing percentage of rats in all groups (four groups) was statistically significant $(P<0.001)$.

The result of Tukey's post hoc test also showed that regardless of the time, the average of wound healing ratio in rats treated in different groups was statistically significant $(P<0.05)$.

The interaction of time and drug treatment in all groups was also statistically significant $(P<0.001)$, meaning that the healing pattern of the wound surface varied during the evaluated time in different treatment groups. So that the gradient of healing of the wound surface in rats was different in all four drug groups and at different evaluation time.

By reviewing the results use of sarcocolla with appropriate time can lead to a faster and significant wound healing in diabetic animals (see Figure 1).

\section{Discussion}

Diabetic ulcers are significant medical problems, which lead to morbidity and mortality in patients with diabetes. Non-healing wounds are responsible for $80 \%-85 \%$ of lower extremity amputation. Since it is a major problem, finding an optimal improving approach is critical (24). The pathological conditions such as abnormal angiogenesis, hypoperfusion, neuronal ischemia and continued trauma result in wound healing failure. Optimal wound healing needs a well-arranged integration of inflammatory process, biochemical mediators, and remodeling process $(5,6,24)$.

Astragalus species exhibited anti-diabetic effect through different active ingredients including polysaccharides, saponins, and flavonoids (25). A. fasciculifolius from the family of Fabaceae and the genus Astragalus, possesses various pharmacological properties and has been used in the conditions such as cold, joint pains, tooth ache and wound healing (gum, flower, root) (26). A. fasciculifolius as a herbal medicine also has immune regulatory functions (27). Moreover, A. fasciculifolius could be administered in

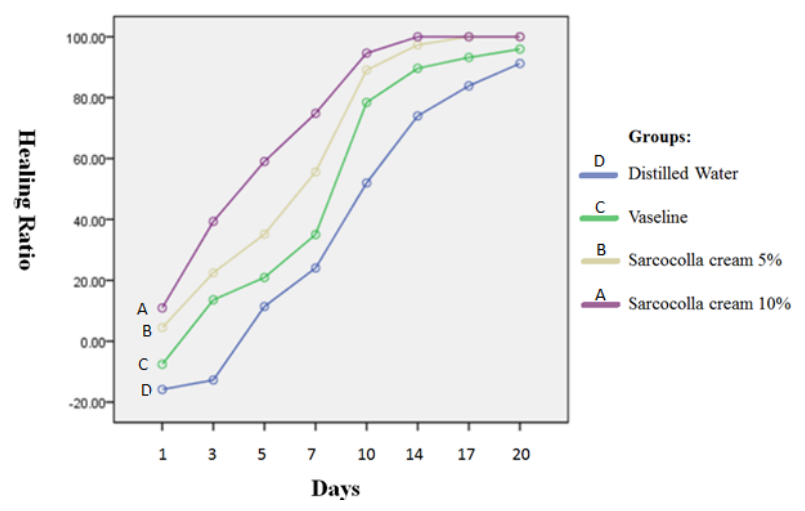

Figure 1. Ratio of wound healing in diabetic rats for different treatments at each evaluation time. other conditions such as fatigue, tightening bone fractures and diabetes (28). As traditional medicine is used commonly in treatment of different disorders in Iran and in other hand, as there is not enough data on the effects of A. fasciculifolius on diabetic wound healing, the purpose of our study was to evaluate the effect of sarcocolla on diabetic wounds of rats.

We observed that cream containing 5\% sarcocolla and $10 \%$ sarcocolla exerts useful effects on the wound-healing ratio. The statistical comparisons revealed that the rate of wound healing in diabetic rats treated with $5 \%$ sarcocolla and $10 \%$ sarcocolla was higher than those treated with petrolatum or distilled water. We showed that sarcocolla had favorable effects on the wound-healing ratio especially during long-term (14 days) at concentrations of $5 \%$ sarcocolla and $10 \%$ sarcocolla. Therefore, the sarcocolla efficacy is more related to the treatment time than with treatment dosage, because we did not find any significant difference in wound-healing ratio between 5\% sarcocolla -7 day and $10 \%$ sarcocolla-7 day groups and between $5 \%$ sarcocolla-14 day and 10\% sarcocolla-14 day groups $(P>$ 0.05 ). Our result is in line with previous studies indicating the protective effects of sarcocolla and other species of Astragalus genus on wound repairing in none diabetic subjects $(14,29)$.

Evidence showed that the Astragalus species via different mechanisms including suppressing inflammation, cell cycle acceleration and improving the secretion of repair factors exerts protective effects on wound healing process (29) and future studies are needed to elucidate the exact mechanism of action of A. fasciculifolius on diabetic wound healing. Moreover, other experimental and clinical studies are recommended in order to analyze the possible adverse effects, toxicity or allergic reactions after topical/ systemic use of $A$. fasciculifolius.

\section{Conclusion}

We showed that $A$. fasciculifolius might be beneficial in the treatment of diabetic wounds. Further studies are needed to clarify the possible mode of action and safety.

\section{Authors' contributions}

SY, FA and AF had contribution in diabetic induction and taking care of animals during the study. FK, FH and MB had role in cream preparation and administration to animals as well as data collection and analysis. $\mathrm{MH}$ and $\mathrm{HJN}$ were advisers in diabetic ulcer induction in animals because of their experience and participated in preparing the related proposal. MM designed the main idea and supervised the experiment. $\mathrm{MH}$ and MAR had contribution in writing the manuscript and journal submission. All read and confirmed the final version of the manuscript.

\section{Conflict of interests}

There is no conflict of interest associated with this study. 


\section{Ethical considerations}

Ethical issues including plagiarism, falsification, misconduct, data fabrication, double publication or redundancy have been carefully observed by authors. The ethical committee of Rafsanjan University of Medical Sciences confirmed the protocol and the ethical code is as follow IR.RUMS.REC.1397.078.

\section{Funding/Support}

This research was supported by the Vice Chancellor for Research and Technology, Rafsanjan University of Medical Sciences, Rafsanjan, Iran (grant No. 97147).

\section{References}

1. Zheng Y, Ley SH, Hu FB. Global aetiology and epidemiology of type 2 diabetes mellitus and its complications. Nat Rev Endocrinol. 2018;14(2):8898. doi: 10.1038/nrendo.2017.151.

2. Ramachandran A. Know the signs and symptoms of diabetes. Indian J Med Res. 2014;140(5):579.

3. Pinhas-Hamiel O, Zeitler P. Acute and chronic complications of type 2 diabetes mellitus in children and adolescents. Lancet. 2007;369(9575):1823-31.

4. Baltzis D, Eleftheriadou I, Veves A. Pathogenesis and treatment of impaired wound healing in diabetes mellitus: new insights. Adv Ther. 2014;31(8):817-36. doi: 10.1007/s12325-014-0140-x.

5. Johnson KE, Wilgus TA. Vascular endothelial growth factor and angiogenesis in the regulation of cutaneous wound repair. Adv Wound Care (New Rochelle). 2014;3(10):647-61. doi: 10.1089/wound.2018.0796.

6. Galkowska H, Wojewodzka U, Olszewski WL. Chemokines, cytokines, and growth factors in keratinocytes and dermal endothelial cells in the margin of chronic diabetic foot ulcers. Wound Repair Regen. 2006;14(5):558-65.

7. Rezabakhsh A, Cheraghi O, Nourazarian A, Hassanpour M, Kazemi M, Ghaderi S, et al. Type 2 diabetes inhibited human mesenchymal stem cells angiogenic response by over-activity of the autophagic pathway. J Cell Biochem. 2017;118(6):1518-30. doi: $10.1002 /$ jcb. 25814 .

8. Okonkwo U, DiPietro L. Diabetes and wound angiogenesis. Int J Mol Sci. 2017;18(7):1419.

9. Gibran NS, Jang YC, Isik FF, Greenhalgh DG, Muffley LA, Underwood RA, et al. Diminished neuropeptide levels contribute to the impaired cutaneous healing response associated with diabetes mellitus. J Surg Res. 2002;108 (1):122-8.

10. Alzahrani HA, Bakhotmah BA, Boukraa L. In vitro susceptibility of diabetic wound bacteria to mixtures of honey, Commiphora molmol and Nigella sativa. Open Nutraceut J. 2011;4:172-5. doi: 10.2174/1876396001104010172.

11. Gupta A, Upadhyay NK, Sawhney R, Kumar R. A poly-herbal formulation accelerates normal and impaired diabetic wound healing. Wound Repair and Regeneration. 2008;16(6):784-90.

12. Kavitha KV, Tiwari S, Purandare VB, Khedkar S, Bhosale SS, Unnikrishnan AG. Choice of wound care in diabetic foot ulcer: a practical approach. World J Diabetes. 2014;5(4):546-56. doi: 10.4239/wjd. v5.i4.546

13. Chavoushi SH, Ghabili K, Kazemi A, Aslanabadi A, BabapourS, Ahmedli R, etal. Surgery for gynecomastia in the islamic golden age: Al-Tasrif of Al-Zahrawi (936-1013 AD). ISRN Surg. 2012;2012:934965. doi: 10.5402/2012/934965.

14. Ghahramani DS, Saeidiani S, Mohammadzadeh R, Shoja GM, Asalforoush RA, Akradi L. A comparative study of the healing effects of calendula and Astragalus fasciculifolius aqueous resin extract on rabbit skin wounds. Journal of Large Animal Clinical Science Research. 2010:3(9):51-61.

15. Zhu H, Wang Y, Liu Z, Wang J, Wan D, Feng S, et al. Antidiabetic and antioxidant effects of catalpol extracted from Rehmannia glutinosa (Di Huang) on rat diabetes induced by streptozotocin and high-fat, high-sugar feed. Chin Med. 2016;11:25. doi: 10.1186/ s13020-016-0096-7.

16. Zare-Bidaki M, Rezaein M, Ali-Abasi P. A review on the ethical issues of animal research. J Rafsanjan Univ Med Sci. 2012;11(6): 575-98.

17. Taghipour Z, Hassanipour M, Mohammadian B, Khanjari A, Taghizadeh R, Kaeidi A, et al. The effects of the topical administration of Pistacia vera oil on the second-degree burn model in rats. Pistachio and Health Journal. 2018;1(2): 7-11. doi:10.22123/ PHJ.2018.115685.1003

18. Furman BL. Streptozotocin-induced diabetic models in mice and rats. Curr Protoc Pharmacol. 2015;70(1):5-47.

19. Nazari M, Hajizadeh MR, Mahmoodi M, Mirzaei MR, Hassanshahi G. The regulatory impacts of Morus alba leaf extract on some enzymes involved in glucose metabolism pathways in diabetic rat liver. Clin Lab. 2013;1;59(5-6):497-504.

20. Tombulturk FK, Kasap M, Tuncdemir M, Polat E, Sirekbasan S, Kanli A, et al. Effects of Lucilia sericata on wound healing in streptozotocin-induced diabetic rats and analysis of its secretome at the proteome level. Hum Exp Toxicol. 2018;37(5):508-20. doi: 10.1177/0960327117714041.

21. Asadi SY, Parsaei P, Karimi M, Ezzati S, Zamiri A, Mohammadizadeh F, et al. Effect of green tea (Camellia sinensis) extract on healing process of surgical wounds in rat. Int J Surg. 2013;11(4):332-7.

22. Kant V, Gopal A, Pathak NN, Kumar P, Tandan SK, Kumar D. Antioxidant and anti-inflammatory potential of curcumin accelerated the cutaneous 
wound healing in streptozotocin-induced diabetic rats. Int Immunopharmacol. 2014;20(2):322-30.

23. Gulcan E, Kuçuk A, Çayci K, Tosun M, Emre H, Koral L, et al. Topical effects of nebivolol on wounds in diabetic rats. Eur J Pharm Sci. 2012;29;47(2):451-5.

24. Hinchliffe R, Valk G, Apelqvist J, Armstrong D, Bakker K, Game F, et al. A systematic review of the effectiveness of interventions to enhance the healing of chronic ulcers of the foot in diabetes. Diabetes Metab Res Rev. 2008;24(S1):119-4441.

25. Kai Z, Michela P, Antonio P, Annamaria P. Biological active ingredients of traditional Chinese herb Astragalus membranaceus on treatment of diabetes: a systematic review. Mini Rev Med Chem. 2015;15(4):315-29. doi: 10.2174/13895575156661502 27113431.

26. Mosaddegh M, Naghibi F, Moazzeni H, Pirani A, Esmaeili S. Ethnobotanical survey of herbal remedies traditionally used in Kohghiluyeh va Boyer Ahmad province of Iran. J Ethnopharmacol. 2012;141(1):8095.

27. Azemi M, Ghafoorian Borougerdnia M, Namjooyan F, Saeidian H, Naanaei SY, Hemmati A. Evaluation of hydroalcoholic extract of Astragalus fasciculifolius Boiss. on immunological factors IFN- $\gamma$, IL-4 in early sensitized mice induced by ovalbumin. Planta Med. 2011;77(12):PM137. doi: 10.1055/s-0031-1282895.

28. Safa O, Soltanipoor MA, Rastegar S, Kazemi M, Dehkordi KN, Ghannadi A. An ethnobotanical survey on Hormozgan province, Iran. Avicenna J Phytomed. 2013;3(1):64-81.

29. Zhao B, Zhang X, Han W, Cheng J, Qin Y. Wound healing effect of an Astragalus membranaceus polysaccharide and its mechanism. Mol Med Rep. 2017;15(6):4077-83. doi: 10.3892/mmr.2017.6488. 